CASE REPORT

\author{
A. Poretti \\ U. Brehmer \\ I. Scheer \\ V. Bernet \\ E. Boltshauser
}

\section{Prenatal and Neonatal MR Imaging Findings in Oral-Facial-Digital Syndrome Type VI}

\begin{abstract}
SUMMARY: We report prenatal and neonatal neuroimaging findings in a case of oral-facial-digital syndrome type VI (OFDS VI). Prenatal MR imaging at 29 weeks' gestation showed hypoplastic cerebellar vermis and hemispheres, the molar tooth sign, and a hypothalamic hamartoma. Neonatal MR imaging confirmed these findings. The neonate developed breathing abnormalities and exhibited frontal bossing, multiple bucco-alveolar frenula, and postaxial hexadactyly of both hands. If the molar tooth sign and a hypothalamic hamartoma are present, prenatal diagnosis of OFDS VI is possible.
\end{abstract}

$\mathbf{0}$ ral-facial-digital syndrome type VI (OFDS VI) or VáradiPapp syndrome (OMIM 277170) is a rare autosomal recessive syndrome $e^{1,2}$ belonging to Joubert syndrome and related disorders (JSRD), ${ }^{3}$ whereof less than 40 cases have been reported. The genetic basis remains unknown. JSRD and OFDS VI can present with similar neurologic symptoms such as hypotonia, delayed milestones, ataxia, cognitive impairment, irregular breathing in the neonatal period, and ocular motor apraxia ${ }^{4}$ as well as facial dysmorphic signs. ${ }^{5}$ However, postaxial polydactyly is a typical finding in OFDS VI but is only rarely observed in JSRD. ${ }^{3}$ Furthermore, tongue hamartoma, bucco-alveolar frenula, or both, are exclusive for OFDS VI. ${ }^{3}$ Characteristic neuroradiologic hallmarks of JSRD include cerebellar vermis hypoplasia in combination with the "molar tooth sign" (MTS), a complex malformation of the midbrain characterized by thickened and elongated superior cerebellar peduncles and an abnormally deep interpeduncular fossa. ${ }^{6}$ These findings are also found in OFD VI. ${ }^{6} \mathrm{Hy}-$ pothalamic hamartoma $(\mathrm{HH})$ is a specific, but not consistently present, feature of OFDS VI. ${ }^{7}$

With the advent of MR imaging, the diagnosis of JSRD can be made prenatally before 24 weeks' gestation. ${ }^{8}$ In this report, we describe prenatal and neonatal MR findings in OFDS VI.

\section{Case Report}

A boy was born at 38 weeks' gestation to a 34-year-old mother. He was the second child of nonconsanguineous and healthy Swiss parents. A routine fetal sonography in week 28 revealed macrocephaly and hypoplasia of the cerebellar vermis. Therefore, a fetal MR imaging examination was performed at 29 weeks' gestation. This showed hypoplastic cerebellar vermis and hemispheres (Fig $1 A$ and $B$ ), enlarged posterior fossa, thickened and elongated superior cerebellar peduncles, corresponding to a MTS (Fig 1A), and a hypothalamic mass representing HH (Fig $1 A-C$ ). On the basis of these findings, the working diagnosis was JS or JSRD. The boy was born by cesarean delivery because of breech presentation and macrocephaly. Apgar scores were 7, 8, and 9 at 1, 5, and 10 minutes, respectively. Birth weight was $3850 \mathrm{~g}$ (90th-95th percentile), length 50 $\mathrm{cm}$ (25th-50th percentile), and head circumference $39.5 \mathrm{~cm}(3.8 \mathrm{~cm}$ above 97 th percentile). The neonate presented with frontal bossing, lowset ears, flat nasal bridge, multiple bucco-alveolar frenula, and postaxial hexadactyly of both hands. Neurologic examination showed limb mus-

Received December 14, 2007; accepted after revision December 30.

From the Departments of Pediatric Neurology (A.P., E.B.), Diagnostic Imaging (U.B., I.S.), and Neonatology and Intensive Care (V.B.), University Children's Hospital of Zurich, Zurich, Switzerland

Please address correspondence to Eugen Boltshauser, Department of Pediatric Neurology, University Children's Hospital, Steinwiesstr 75, 8032 Zurich, Switzerland; e-mail: Eugen.Boltshauser@kispi.uzh.ch

DOI 10.3174/ajnr.A1038 cular hypertonia. In the first hours, he developed recurrent respiratory patterns of alternating tachypnea with respiratory rates up to 200 and apnea followed by bradycardia. MR imaging on day 2 revealed cerebellar vermis agenesis, cerebellar hemispheric hypoplasia, an enlarged fourth ventricle and fossa posterior, elongated and thickened superior cerebellar peduncles, deep interpeduncular fossa corresponding to an MTS (Fig $2 A$ and $B)$, and an $\mathrm{HH}(\mathrm{Fig} 2 A-C)$. The diagnosis of OFDS VI was made. The boy needed a few mechanical resuscitations for severe apneas and bradycardia. Because of the poor prognosis and the repeated severe apneas, the decision was made, together with the parents, that the boy not be resuscitated anymore. He died at the age of 10 weeks at home.

\section{Discussion}

Fetal MR imaging can be very helpful in the diagnosis of posterior fossa abnormalities ${ }^{9}$ and permits superior discrimination and resolution of the different anatomic structures compared with sonography. Doherty et $\mathrm{al}^{8}$ reported prenatal MR findings of a fetus with a risk for JSRD at 21 weeks' gestation. MR imaging revealed a very small and rostrally located vermis and, inferiorly, a connection between the fourth ventricle and fossa posterior. However, a typical MTS was not seen. Fluss et $\mathrm{al}^{10}$ reported a prenatal diagnosis of JS at 27 weeks' gestation with identification of a typical MTS. In the case of our patient, the MTS was also identifiable at 29 weeks' gestation. Therefore, fetal MR imaging can identify the MTS from 27 weeks' gestation.

Two articles reported prenatal sonography findings of OFDS VI such as polydactyly and abnormalities in the posterior fossa, but prenatal MR imaging was not performed. ${ }^{11,12}$ Prenatal MR findings of OFDS VI have not been reported previously. Neuroimaging features of OFDS VI include cerebellar vermis hypoplasia and the MTS, whereas HH is occasionally found. However, most cases of $\mathrm{HH}$ are sporadic, and in approximately $5 \%$ of cases, $\mathrm{HH}$ is a finding of Pallister-Hall syndrome. ${ }^{13}$ On MR imaging, $\mathrm{HH}$ appears isointense compared with gray matter on T1weighted sequences and isointense to slightly hyperintense on T2-weighted sequences. ${ }^{14}$ Prenatal diagnosis of nonsyndromic $\mathrm{HH}$ had been reported in 1 patient at 26 weeks' gestation. ${ }^{15}$ In the case of our patient, $\mathrm{HH}$ was identifiable at 29 weeks' gestation.

In conclusion, a prenatal diagnosis of OFDS VI is possible with fetal MR imaging from 29 weeks' gestation. Therefore, if prenatal MR imaging shows findings compatible with JSRD, an $\mathrm{HH}$ should be sought. However, because of the rarity of the disease, a prenatal diagnosis of OFDS VI can be easily missed. More cases have to be identified to further characterize the chronologic appearance of the neuroimaging features of OFDS VI. 

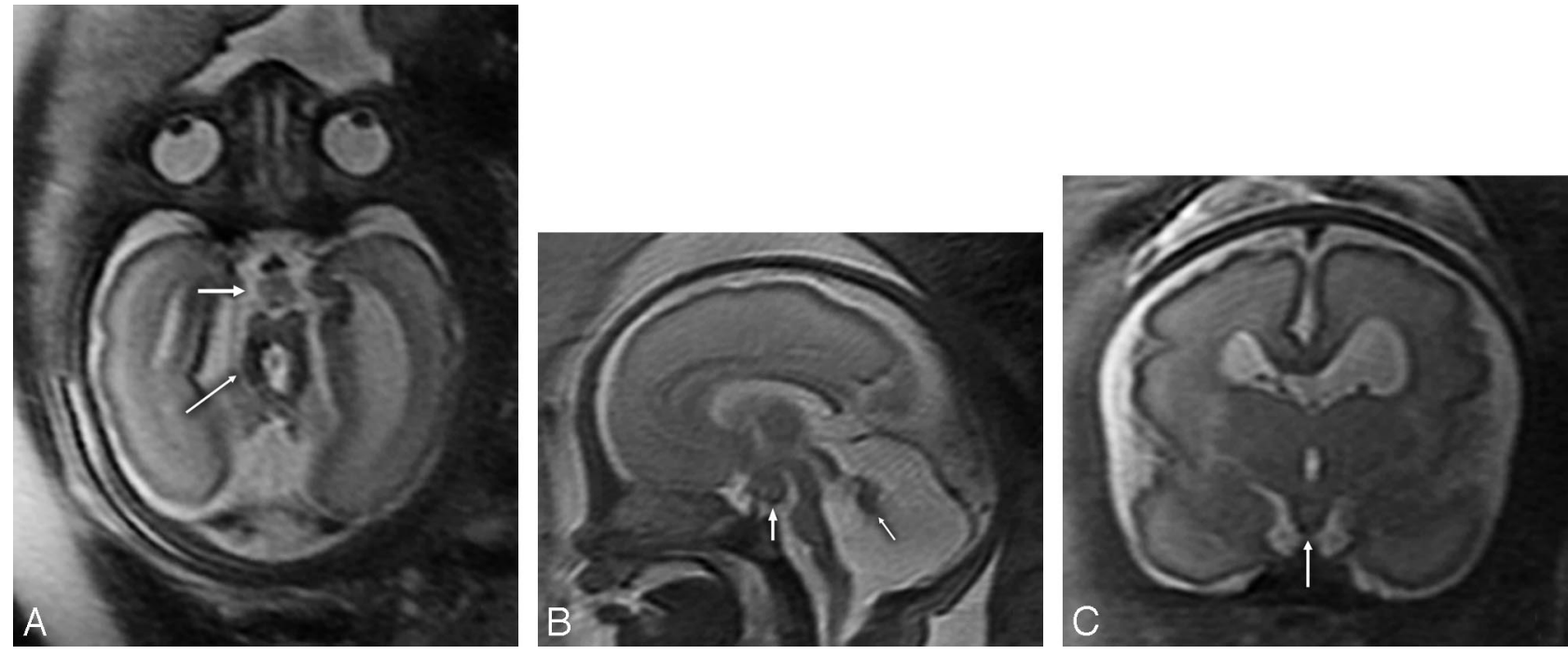

Fig 1. Fetal T2-weighted MR imaging at 29 gestational weeks. A, Axial MR image showing hypoplasia of both cerebellar hemispheres, the characteristic MTS (thin arrow) with thickened and elongated superior cerebellar peduncles and an abnormally deep interpeduncular fossa, and an HH (thick arrow). B, Sagittal MR image revealing significant hypoplasia of the cerebellar vermis (arrow), an enlarged fourth ventricle and posterior fossa, an $\mathrm{HH}$ (white arrow), and a thin brain stem. C, Coronal MR image demonstrating an HH (white arrow)
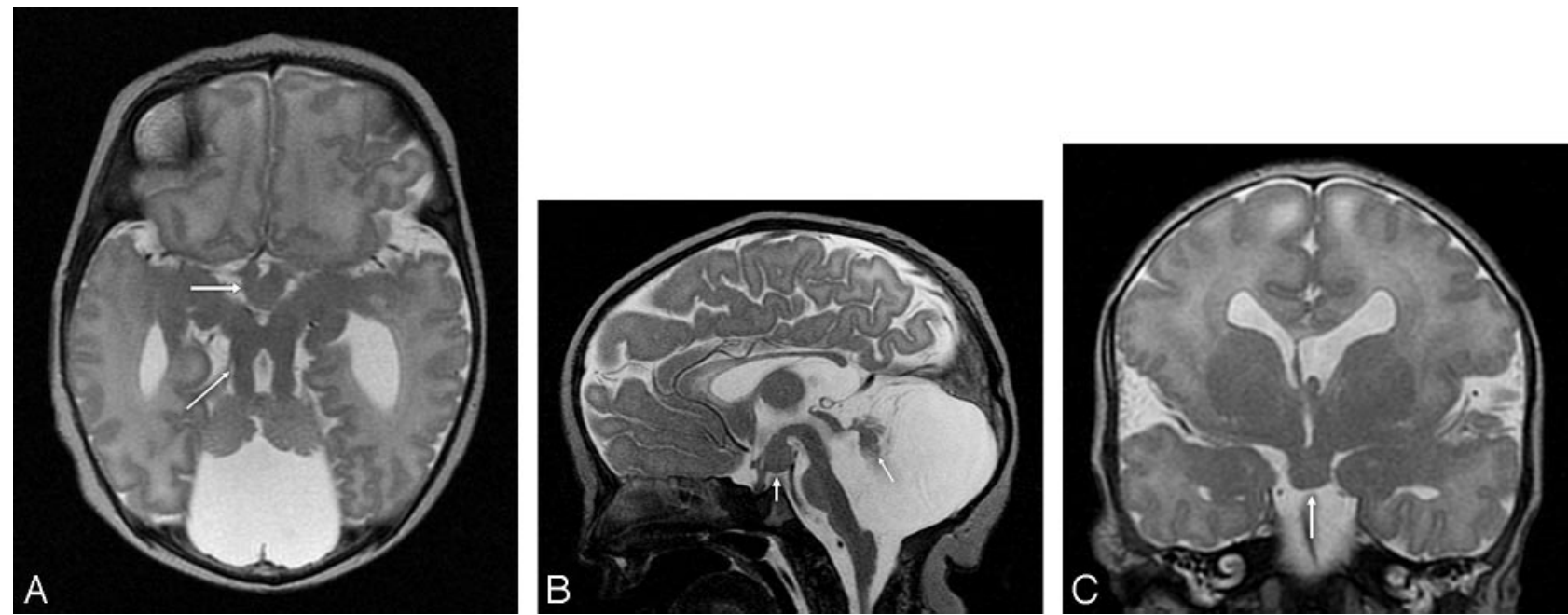

Fig 2. T2-weighted MR imaging at 2 days of age. A, Axial MR image showing hypoplasia of the vermis and both cerebellar hemispheres, the characteristic MTS (thin arrow) with thickened and elongated superior cerebellar peduncles, an abnormally deep interpeduncular fossa, and an HH (thick arrow). B, Sagittal MR image revealing significant hypoplasia of the cerebellar vermis and dysplasia of the remnants of the cerebellar vermis (arrow), an enlarged fourth ventricle and posterior fossa, and an HH (white arrow). In addition, the corpus callosum and the brain stem are hypoplastic, the pituitary stalk is thick, and the interthalamic adhesion is large. C, Coronal MR image demonstrating an HH (arrow), a missing left leaf of the septum pellucidum, a bulky left fornix, and a probably dysplastic cerebral cortex in the left Sylvian fissure.

\section{Acknowledgments}

We thank Beth Padden, MD, for help in editing the manuscript. Dr. Poretti was financially supported by a donation from the United Bank of Switzerland. This donation was made at the request of an anonymous client.

\section{References}

1. Váradi V, Szabo L, Papp Z. Syndrome of polydactyly, cleft lip/palate or lingual lump, and psychomotor retardation in endogamic gypsies. J Med Genet 1980;17:119-22

2. Munke M, McDonald DM, Cronister A, et al. Oral-facial-digital syndrome type VI (Váradi syndrome): further clinical delineation. Am J Med Genet 1990;35:360-69

3. Parisi MA, Doherty D, Chance PF, et al. Joubert syndrome (and related disorders) (OMIM 213300). Eur J Hum Genet 2007;15:511-21

4. Maria BL, Boltshauser E, Palmer SC, et al. Clinical features and revised diagnostic criteria in Joubert syndrome. J Child Neurol 1999;14:583-90

5. Braddock SR, Henley KM, Maria BL. The face of Joubert syndrome: a study of dysmorphology and anthropometry. Am J Med Genet A 2007;143:3235-42

6. Gleeson JG, Keeler LC, Parisi MA, et al. Molar tooth sign of the midbrainhindbrain junction: occurrence in multiple distinct syndromes. Am J Med Genet A 2004;125:125-34
7. Stephan MJ, Brooks KL, Moore DC, et al. Hypothalamic hamartoma in oral-facialdigital syndrome type VI (Váradi syndrome). Am J Med Genet 1994;51:131-36

8. Doherty D, Glass IA, Siebert JR, et al. Prenatal diagnosis in pregnancies at risk for Joubert syndrome by ultrasound and MRI. Prenat Diagn 2005;25:442-47

9. Adamsbaum C, Moutard ML, Andre C, et al. MRI of the fetal posterior fossa Pediatr Radiol 2005;35:124-40

10. Fluss J, Blaser S, Chitayat D, et al. Molar tooth sign in fetal brain magnetic resonance imaging leading to the prenatal diagnosis of Joubert syndrome and related disorders. J Child Neurol 2006;21:320-24

11. Guven MA, Ceylaner S, Prefumo F, et al. Prenatal sonographic findings in a case of Varadi-Papp syndrome. Prenat Diagn 2004;24:989-91

12. McPherson E, Zaleski C, Mascola M. Prenatal diagnosis of episodic tachypnea in an infant with OFD VI. Am J Med Genet A 2006;140:2146-49

13. Kang S, Graham JM, Jr., Olney AH, Biesecker LG. GLI3 frameshift mutations cause autosomal dominant Pallister-Hall syndrome. Nat Genet 1997;15:266-68

14. Freeman JL, Coleman LT, Wellard RM, et al. MR imaging and spectroscopic study of epileptogenic hypothalamic hamartomas: analysis of 72 cases. AJNR Am J Neuroradiol 2004;25:450-62

15. Booth TN, Timmons C, Shapiro K, et al. Pre- and postnatal MR imaging of hypothalamic hamartomas associated with arachnoid cysts. AJNR Am J Neuroradiol 2004;25:1283-85 